RAD Conference Proceedings, vol. 5, pp. 28-31, 2021

ISSN 2466-4626 (online) | DOI: 10.21175/RadProc.2021.05

www.rad-proceedings.org

\title{
MULTIPLE THROMBOSES AS LATE COMPLICATIONS IN A PATIENT WITH PNEUMONIA CAUSED BY COVID-19 INFECTION
}

\begin{abstract}
Mitko Mitev*
Department of Medical Physics, Biophysics, Roentgenology and Radiology, Faculty of Medicine, Department of Diagnostic Imaging, University Hospital ‘St. Kirkovich', Trakia University - Stara Zagora, Stara Zagora, Bulgaria
\end{abstract}

\begin{abstract}
Introduction. Venous and arterial thromboembolism is associated with COVID-19, but there are few studies of lower limb ischemia as a later complication of infection. The study presents identified early and late complications caused by COVID-19, with the presence of multiple thromboses in the aorta and peripheral vessels. Materials and methods. A patient is an 8o-year-old man hospitalized with COVID-19 (SARS-CoV-2). The study was conducted in 2021. MDCT Siemens Definition AS was used. A computed tomography angiography was made with Omnipaque, $100 \mathrm{ml}$. The data was processed with Syngo.via workstation and VB4OB_NFO2 software version.

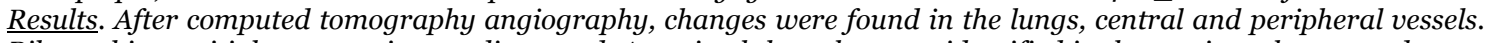
Bilateral interstitial pneumonia was diagnosed. A parietal thrombus was identified in the aortic arch area and acute thrombosis on the left side of the common iliac artery and the right side of the superficial femoral artery as an acute later complication of the infection. The patient was sent for emergency surgery to remove the found blood clots in the lower limbs. Conclusion. The application of the correct diagnostic algorithm in this clinical case with the application of CT scan with contrast helped to quickly identify early complications and to avoid more severe and later changes both in the vessels and in other organs and systems.
\end{abstract}

Key words: Aorta, Arterial thrombosis, complications, COVID-19, interstitial pneumonia

\section{INTRODUCTION}

Arterial and venous thromboses have been reported as later complications in hospitalized patients with COVID-19 [1]-[5]. Coagulation disorders with an increased risk of venous thrombosis and pulmonary embolism have been reported in $4.4-8.2 \%$ of patients with COVID-19 [1], [6]-[8]. Studies of venous and arterial thrombosis have been shown to be extremely important in patients with COVID-19 [5]. The present study presents identified early and late complications caused by COVID-19, with the presence of multiple thromboses in the aorta and peripheral vessels.

\section{MATERIALS AND METHODS}

A patient is an 80-year-old man hospitalized with COVID-19 (SARS-CoV-2). The study was conducted in 2021. MDCT Siemens Definition AS was used. A computed tomography angiography was made with Omnipaque, $100 \mathrm{ml}$. The data was processed with Syngo.via workstation and VB40B_NFo2 software version.

\section{RESULTS - CASE REPORT}

It was about a patient, a man of 80 years, who during a visit to a pulmonologist reported general fatigue, lethargy, and the appearance of progressive dry cough for 10 days, suffocation and a temperature of 37.6 degrees. The described symptoms suggested a viral infection with COVID-19. The rapid antigen test and PCR test performed were positive. The patient was hospitalized urgently in the pneumology clinic. As comorbidity, the patient had proven hypertension - II cardiac form. The chest radiography revealed bilateral changes of frosted glass type and the presence of a striped-mesh-like interstitial pattern, mainly subpleural, apical and basal, with a more pronounced dominance of the changes on the right. In conclusion, it was interpreted as bilateral infiltrative-inflammatory changes with a predominance of the interstitial component caused by COVID-19 SARS-CoV-2) infection. Paraclinical studies showed high values of CRP187 mg/L, lactate dehydrogenase (LDH ) $815 \mathrm{U} / \mathrm{l}$, glucose $7.4 \mathrm{mmol} / \mathrm{l}$ and low oxygen saturation - 88\%. Antibiotic treatment was started together with other treatments, in which the patient's condition significantly improved. During the clinical stay, the patient reported severe pain in both lower limbs and after consultation with a vascular surgeon, no pulsation in the right lower limb was found. The Computed tomography angiography (CTA; Omnipaque $100 \mathrm{ml}$, with Syngo.via workstation and VB4OB_NFO2 software version) revealed changes in the lungs, central and peripheral vessels. In the area of the thorax, bilaterally, the lung parenchyma was unfolded. The trachea, main and segmental bronchi were freely passable. The

*mitev.mitko69@gmail.com 
truncus pulmonalis, the main and peripheral pulmonary vessels, had the correct architectonics, with no defects in the filling. Frosted glass-type changes, striped-mesh-like interstitial compaction and deformed interstitium with blurred outlines, small areas of parenchyma consolidation located subpleural and more pronounced changes on the right side were found. The hill was hypervolemic with proper structure. The pleural spaces were free (Figure 1).

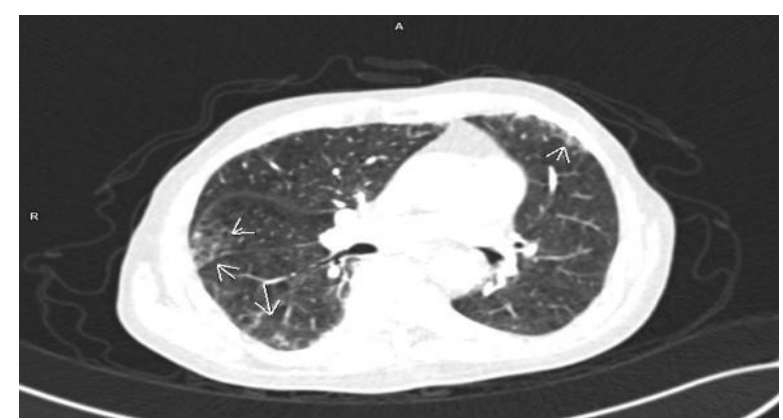

Figure 1. Changes in the lung parenchyma in interstitial infiltrative inflammatory changes correlating with Covid 19 infection

From the central vessels, the ascending aorta was generally elongated without aneurysmically dilated areas and defects in the filling. In the area of the aortic arch, a wall defect was found in the filling with axial dimensions of $7 / 8 \mathrm{~mm}$ (Figure 2).

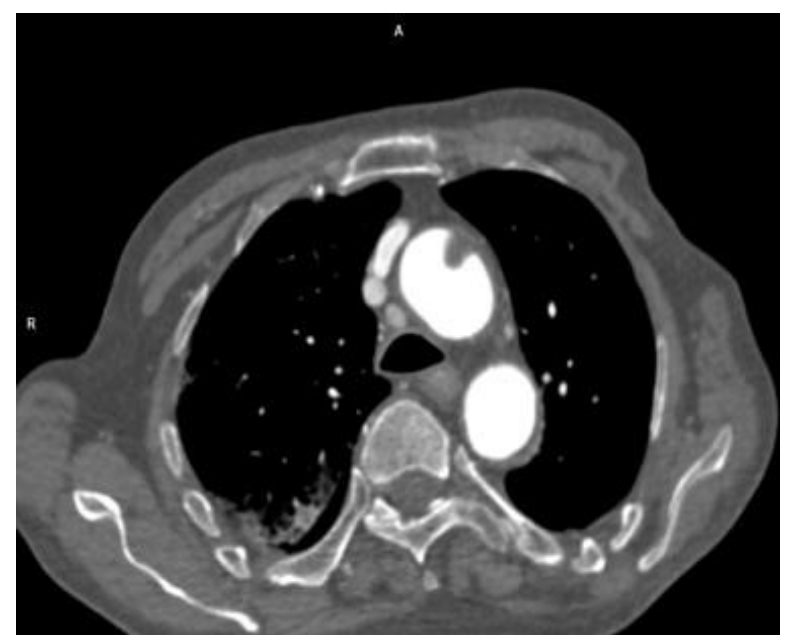

Figure 2. Wall thrombus in the area of the aortic arch

The thoracic and abdominal parts had small wall defects of atheromatous plaques and homogeneous filling with a contrast of the lumen. On the left, the common iliac artery was transversely abruptly interrupted. No contrast material was observed distally in the external iliac artery and the vessels of the limb. On the right, the iliac vessels were elongated with wall defects, but were traced along their entire length. The right superficial femoral artery was obliterated from its exit to the lower third. The right popliteal artery was normal in size and imaging. The crural arteries on the right were traced along their entire length but were smaller in size due to the reduced blood flow (Figure 3).

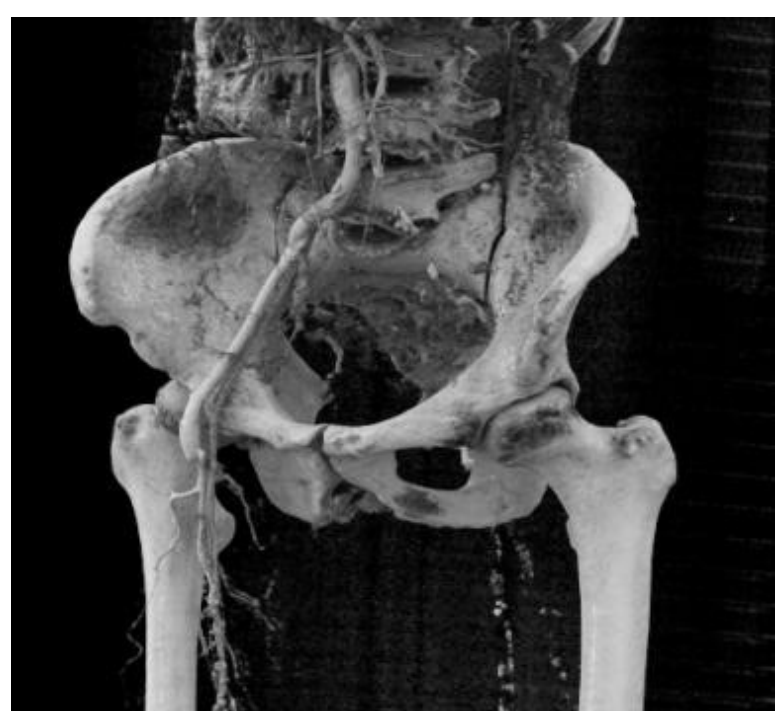

Figure 3. 3D image (reconstruction) depicting thrombosis of the right superficial femoral artery and the left common iliac artery (trombosis artetia femoralis superficialri dextra et arteria iliaca communis sinistra)

In conclusion, it was found that the case referred to bilateral infiltrative-inflammatory changes with the presence of a predominance of the interstitial component and the availability of initial areas with alveolar changes as a result of COVID-19 infection. Elongation of the ascending aorta was found. Presence of a wall thrombus in the area of the aortic arch and acute thrombosis on the left of the common iliac artery and on the right of the superficial femoral artery as an acute complication as a result of established infection with proven COVID-19. The patient was sent for emergency surgery to remove established blood clots in the lower limbs.

\section{DisCUSSION}

As a result of proven COVID-19 and the correct algorithm of work with the complex study of physical, paraclinical and imaging methods, the study revealed bilateral interstitial pneumonia caused by the COVID19 virus. Properly performed antibiotics and other treatments quickly and significantly improved the changes caused by lung pneumonia. The performed CT angiography showed a number of thromboses in the central and peripheral vessels that were proven to be an acute complication directing the patient to prompt surgical intervention to remove the thrombosis. The COVID-19 infection can provoke venous and arterial thromboembolism due to excessive inflammation, hypoxia, immobility, and disseminated intravascular coagulation, but there have been few reports of lower limb ischemia as a major manifestation of the disease [9]. Costa et al. [9] described a case of a 69-year-old COVID-19-positive patient in whom arterial embolization of both limbs, the right internal iliac artery, and the superior mesenteric artery ware identified by a CTA. Patients infected with COVID-19 are at risk of arterial thromboembolism [9]. The study of complications in such patients is crucial for the development of specific prevention protocols [9]. 
According to Cheruiyot et al. [10] the severe form of coronavirus infectious disease is mediated in part by a hypercoagulable condition characterized by micro- and macrovascular thrombotic angiopathy. They point out that while venous thrombosis in such patients is well described, data on arterial thrombosis are still insufficient. After summarizing the scientific literature, Cheruiyot et al. [10] find that arterial thrombosis occurs in about $4 \%$ of severely ill patients with COVID19 and is often symptomatic and can affect multiple arteries. The authors point out that it is necessary to study the mechanism of arterial thrombosis in COVID19 infections in order the treatment outcome to be favourable. Goldmann et al. [11] investigate whether arterial thrombosis of the lower limbs is associated with COVID-19 and whether it is more severe in patients with this infection. The authors conclude that COVID-19 infection is associated with arterial thrombosis of the lower limbs, characterized by a greater accumulation of clots and a worse prognosis. Schweblin et al. [5] report a case in a 54-year-old man with acute arterial ischemia of the lower right limb, identified by CT angiography, due to multistage arterial thrombosis, which they present as a late manifestation of COVID-19. According to the authors, a better understanding of the coagulopathy in COVID-19 is essential for the prevention and treatment of venous and arterial thromboembolic conditions. The mean time reported in the scientific literature identified between the COVID-19 symptoms and the appearance of arterial thrombosis is 14 days, indicating delayed complications caused by COVID-19 [5].

Virtual bronchoscopy detects changes that guide the clinician's behaviour and treatment algorithm. We suggest emergency surgery for thromboses that affect the large vessels, as well as the prescription of conservative treatment with anticoagulant and antiplatelet therapy according to a scheme at home to prevent the formation of subsequent thromboses. We recommend dynamic monitoring of the therapy and the patient's condition.

\section{CONCLUSION}

Early complications caused by COVID-19 are bilateral interstitial infiltrative-inflammatory changes. As a result of the correct algorithm and behaviour in clinical manifestations, the patient was examined by CT angiography and many thromboses in the central and peripheral vessels were found as late complications. This helped to conduct both proper antibiotic and other lung treatment and accurate and rapid detection of late complications and subsequent acute vascular thrombosis, directing the patient to prompt surgery to remove thrombosis in the identified areas and restore the proper blood circulation. It wasfound, as in many other cases, that COVID-19 infection caused multiorgan changes and many acute and late subsequent complications. Applying the correct diagnostic algorithm in this clinical case with the application of CT scan with contrast helped to quickly identify early complications and to avoid more severe and later changes both in the vessels and in other organs and systems.
Acknowledgements: The authors would like to thank the Trakia University - Stara Zagora, the Republic of Bulgaria, for the funding received under the National Research Program "Young Scientists and PhD Students" and the support provided.

\section{REFERENCES}

1. J. Helms et al., "High risk of thrombosis in patients with severe SARS-CoV-2 infection: a multicenter prospective cohort study," Intensive Care Med., vol. 46, no. 6, pp. 1089-1098, May 2020. https://doi.org/10.1007/s00134-020-06062-x PMid: 32367170

2. P. Vulliamy, S. Jacob, R.A. Davenport, "Acute aortoiliac and mesenteric arterial thromboses as presenting features of COVID-19," Br. J. Haem., vol. 189, no. 6, pp. 1053-1054, May 2020. https://doi.org/10.1111/bjh.16760 PMid: 32353183

3. P. Kaur et al., "Acute upper limb ischemia in a patient with COVID-19," Hematol. Oncol. Stem. Cell. Ther., vol. 14, no. 6, pp. 1658-3876, May 2020. https://doi.org/10.1016/j.hemonc.2020.05.001 PMid: 32405288

4. O. de Barry et al., "Arterial and venous abdominal thrombosis in a 79-year-old woman with COVID-19 pneumonia," Radiol. Case Rep., vol. 15, no. 7, pp. 1054-1057, Apr. 2020.

https://doi.org/10.1016/j.radcr.2020.04.055 PMid: 32351657

5. C. Schweblin, A.L. Hachulla, M. Roffi, F. Glauser, "Delayed manifestation of COVID-19 presenting as lower extremity multilevel arterial thrombosis: a case report," European Heart Journal - Case Reports, vol. 4, no. 6, pp. 1-4, Dec. 2020.

https://doi.org/10.1093/ehjcr/ytaa371 PMid: 33437919

6. J.F. Llitjos et al., "High incidence of venous thromboembolic events in anticoagulated severe COVID-19 patients," J. Thromb. Haemost., vol. 18, no. 7, pp. 743-1746, May 2020.

https://doi.org/10.1111/jth.14869 PMid: 32320517

7. D. Wichmann et al., "Autopsy findings and venous thromboembolism in patients with COVID-19: a prospective cohort study," Ann. Intern. Med., vol. 173, no. 4, pp. 268-277, Aug. 2020. https://doi.org/10.7326/M20-2003 PMid: 32374815

8. P. Fontana et al., "Venous thromboembolism in COVID-19: systematic review of reported risks and current guidelines," Swiss. Med. Wkly., vol. 150, article no. w20301, Jun. 2020.

https://doi.org/10.4414/smw.2020.20301 PMid: 32640479

9. V.O. Costa et al., "Acute arterial occlusion of the lower limb as the main clinicalmanifestation in a patient with Covid-19 - Case Report," International Journal of Surgery Case Reports, vol. 77, pp. 454-458, Nov. 2020.

https://doi.org/10.1016/j.ijscr.2020.11.046 PMid: 33200062

10. I. Cheruiyot et al., "Arterial Thrombosis in Coronavirus Disease 2019 Patients: A Rapid Systematic Review," Ann. Vasc. Surg., vol. 70, pp. 273-281, Aug. 2020. https://doi.org/10.1016/j.avsg.2020.08.087 PMid: 32866574

11. I.A. Goldman, K. Ye, M.H. Scheinfeld, "Lower-extremity Arterial Thrombosis Associated with COVID-19 Is Characterized by Greater Thrombus Burden and 
M. Mitev, Multiple thromboses as late COVID-19 complications, RAD Conf. Proc., vol. 5, 2021, 28-31

Increased Rate of Amputation and Death," Radiol.,

vol. 297, no. 2, pp. E263-E269, Jul. 2020.

https://doi.org/10.1148/radiol.2020202348

PMid: 32673190 